\title{
Symmetry-based reciprocity: evolutionary constraints on a proximate mechanism
}

Marco Campennì, Gabriele Schino

Background. While the evolution of reciprocal cooperation has attracted an enormous attention, the proximate mechanisms underlying the ability of animals to cooperate reciprocally are comparatively neglected. Symmetry-based reciprocity is a hypothetical proximate mechanism that has been suggested to be widespread among cognitively unsophisticated animals. Methods. We developed two agent based models of symmetrybased reciprocity (one relying on an arbitrary tag and the other on interindividual proximity) and tested their ability both to reproduce significant emergent features of cooperation in group living animals and to promote the evolution of cooperation. Results. Populations formed by agents adopting symmetry-based reciprocity showed differentiated "social relationships" and a positive correlation between cooperation given and received, two common aspects of animal cooperation. However, when reproduction and selection across multiple generations were added to the models, agents adopting symmetry-based reciprocity were outcompeted by selfish agents that never cooperated. Discussion. In order to evolve, hypothetical proximate mechanisms must be able to stand competition from alternative strategies. While the results of our simulations require confirmation using analytical methods, we provisionally suggest symmetry-based reciprocity is to be abandoned as a possible proximate mechanism underlying the ability of animals to reciprocate cooperative interactions. 
3 Symmetry-Based Reciprocity: Evolutionary Constraints on a

\section{Proximate Mechanism}

\section{Marco Campenni ${ }^{1,2}$ and Gabriele Schino ${ }^{3, *}$}

7

$8{ }^{1}$ Stockholm Resilience Centre, Stockholms Universitet, Stockholm, Sweden

$9{ }^{2}$ Department of Psychology, Arizona State University, Tempe, AZ, USA

$10{ }^{3}$ Istituto di Scienze e Tecnologie della Cognizione, C.N.R., Rome, Italy

16 * Correspondence to: Gabriele Schino, Istituto di Scienze e Tecnologie della Cognizione,

17 C.N.R., Via Ulisse Aldrovandi 16b, 00197 Roma, Italy; email: g.schino@istc.cnr.it 


\section{ABSTRACT}

22 Background. While the evolution of reciprocal cooperation has attracted an enormous attention,

23 the proximate mechanisms underlying the ability of animals to cooperate reciprocally are

24 comparatively neglected. Symmetry-based reciprocity is a hypothetical proximate mechanism

25 that has been suggested to be widespread among cognitively unsophisticated animals.

26 Methods. We developed two agent based models of symmetry-based reciprocity (one relying on

27 an arbitrary tag and the other on interindividual proximity) and tested their ability both to

28 reproduce significant emergent features of cooperation in group living animals and to promote

29 the evolution of cooperation.

30 Results. Populations formed by agents adopting symmetry-based reciprocity showed

31 differentiated "social relationships" and a positive correlation between cooperation given and

32 received, two common aspects of animal cooperation. However, when reproduction and selection

33 across multiple generations were added to the models, agents adopting symmetry-based

34 reciprocity were outcompeted by selfish agents that never cooperated.

35 Discussion. In order to evolve, hypothetical proximate mechanisms must be able to stand

36 competition from alternative strategies. While the results of our simulations require confirmation

37 using analytical methods, we provisionally suggest symmetry-based reciprocity is to be

38 abandoned as a possible proximate mechanism underlying the ability of animals to reciprocate

39 cooperative interactions. 


\section{INTRODUCTION}

A complete understanding of any biological phenomenon requires addressing four separate (but interacting) aspects: its ontogeny, phylogeny, proximate causation and ultimate function (Mayr, 1961, 1982; Tinbergen, 1963). Nevertheless, it is often the case that only one of these aspects is emphasized, at the expense of the others. The interactions between different aspects are often similarly ignored (Hofmann et al., 2014; Fawcett, Marshall \& Higginson, 2015).

A paradigmatic example is the study of reciprocal cooperation in animals. Given the obvious problem of explaining how cooperative behaviors (i.e., behaviors that benefit other individuals) could be favored by natural selection, the study of the ultimate function and selective mechanisms underlying cooperative behaviors has prevailed in the literature, while other aspects have been systematically neglected. Thus, the role of reciprocity in the evolution of cooperation has been a topic for debate for more than 40 years (Trivers, 1971, 2006) while the study of the proximate mechanisms supporting the ability to reciprocate cooperative interactions has only recently been addressed.

The first to propose a list of possible proximate mechanisms underlying reciprocity were Brosnan \& de Waal (2002). Building on previous work by de Waal (de Waal \& Luttrell, 1986, 1988; de Waal, 2000), they proposed three hypothetical proximate mechanisms: symmetrybased, attitudinal and calculated reciprocity. The latter two mechanisms were later elaborated by Schino \& Aureli (2009, 2010, in press).

Our focus in this paper is on the first of the mechanisms proposed by Brosnan \& de Waal (2002), symmetry-based reciprocity. This was conceived as the simplest and least cognitively demanding of the three and, as such, it was supposed to be widespread in the animal kingdom. 
65 Symmetry-based reciprocity is supposed to operate whenever the choice of the recipient of

66 cooperation is based on symmetrical aspects of the relationships between individuals.

67 Symmetrical aspects of the relationships could include similarity in age or dominance rank or

68 mutual association. This mechanism does not involve any form of score keeping of cooperation

69 received and is therefore cognitively extremely simple. Importantly, given that characteristics

70 such as age similarity or interindividual proximity are by definition symmetrical, the resulting

71 choice of partner is necessarily reciprocal, meaning that if individual $\mathrm{A}$ is a preferred partner of

72 B, B will necessarily be among the preferred partners of A. Symmetry-based reciprocity was thus

73 proposed to explain the positive correlation between cooperation given and received across pairs

74 of individuals that is often observed in group-living animals (see Schino \& Aureli, in press, for a

75 review). Note that the term reciprocity is generally used to refer to any contingent cooperative

76 investment that is based on the cooperative returns (Carter 2014). As such, symmetry-based

77 reciprocity would not fit the definition. Strictly speaking, in symmetry-based reciprocity what is

78 "reciprocal" is the outcome of a process (symmetrical choices) and not a cause (contingency on

79 received cooperation) of the process.

80 Reciprocity of cooperative interactions can in principle result from two different

81 processes, first distinguished by Bull \& Rice (1991): partner fidelity (later called partner control

82 by Noë, 2006) and partner choice. Partner control models conceive dyads of interacting

83 individuals as conceptually isolated, so that the choice to behave cooperatively or not depends

84 only on the past behavior of the partner. Partner choice models include a comparative

85 component, so that individuals chose the partner to which they direct their cooperative behavior

86 on the basis of a comparison of cooperation received (or anyhow available) from the different

87 potential partners. Symmetry-based reciprocity is a hypothetical proximate mechanism that is 
88 clearly part of a partner choice process, since animals are supposed to make their decisions on

89 the basis of a comparison of the available partners (animals are supposed to choose to cooperate

90 with those most similar to themselves). Note, however, that no account of cooperation received

91 is taken.

92 The acceptance of the concept of symmetry-based reciprocity relies on its generating

93 behavior as seen in simple cooperative societies. Biological phenomena, however, require

94 explanation in terms of all of the four interrelated aspects mentioned above. In particular,

95 although proximate causation and ultimate function are logically separate issues, it is clear that

96 any hypothesis addressing one must also be compatible with what is known about the other. The

97 interactions between proximate causation and ultimate function have been most often

98 instantiated in terms of the constraints that the former can impose on the latter (e.g., Stevens \&

99 Hauser, 2004, for reciprocity; Holekamp, Swanson \&van Meter, 2013, for behavioral flexibility;

100 Gould \& Lewontin 1979, for a more general argument). It is our suggestion that the reverse

101 constraint can apply in the case of symmetry-based reciprocity. In other words, we suggest (and

102 test in this paper) that even if symmetry-based reciprocity provides a plausible mechanism

103 explaining how animals behave, it cannot be accepted as a valid explanation because it is not a

104 mechanism that can evolve, that is, natural selection will always eliminate from an evolving

105 population those individuals that behave according to symmetry-based reciprocity.

106 Symmetry-based reciprocity can be considered as belonging to a set of models in which

107 cooperation is directed to "similar" individuals. This is, however, a rather heterogeneous set that

108 includes at least five different subgroups:

109 1. Models of of kin-selected cooperation based on phenotype matching (Rousset \& Roze,

110 2007). In these models, cues used to choose the preferred recipients of cooperation are 
111 genetically determined and identify kin (similarity depends on common descent).

112 Cooperation is ultimately favored by kin selection (Hamilton 1964).

113 2. Models of tag-based cooperation in which the cue (tag) used to identify the preferred

114 recipients of cooperation is genetically determined and also encode cooperation (or

115 cooperation is encoded by closely linked genes). This is the so called "green beard"

116 effect, that is however vulnerable to invasion by mutants that carry the tag but do not

117 cooperate (Riolo, Cohen \& Axelrod, 2001; Lehman \& Keller 2006; Gardner \& West

118 2010).

119 3. Models of tag-based cooperation in which the cue (tag) used to identify the preferred

120 recipients of cooperation is genetically determined and does not encode cooperation. In

121 these models, cooperation cannot generally evolve unless the population is highly

122 structured, leading to preferential interactions between kin and, ultimately, kin-selected

123 cooperation (Hammond \& Axelrod, 2006a, 2006b).

124 4. Models of tag-based cooperation in which the cue (tag) used to identify the preferred recipients of cooperation is cooperativeness itself. These are models of indirect reciprocity based on reputation or competitive altruism. They show cooperation can evolve, provided the tag used to identify preferred recipients is indeed informative of the recipient's behavior (Roberts, 1998; Nowak \& Sigmund, 2006).

5. Models of tag-based cooperation in which the cue (tag) used to identify the preferred recipients of cooperation is not genetically determined. This is true symmetry-based reciprocity where tags can include characteristics such as age or dominance rank.

133 above subgroups of models. While the first four subgroups above have attracted a great deal of 
134 attention, symmetry-based reciprocity has been somewhat neglected by theoreticians. In this

135 study, we tested the hypothesis (detailed above) that symmetry-based reciprocity cannot evolve

136 by developing a set of agent-based models of symmetry-based reciprocation of cooperative

137 interactions. First, we tested whether groups of agents adopting a strategy of symmetry-based

138 reciprocation do reproduce features of the distribution of cooperative behavior observed among

139 group living animals, i.e., if symmetry-based reciprocity can indeed result in the phenomenon it

140 was originally conceived to explain. Second, we tested whether agents adopting symmetry-based

141 reciprocation are evolutionarily successful against selfish agents that do not cooperate.

142

143 MATERIALS \& METHODS

144 Agent-based models were implemented using the NetLogo platform (NetLogo 5.0.5;

145 Wilensky, 1999). Statistical analyses were conducted in R, version 2.14.2 (R Development Core

146 Team, 2012). Social Network Analyses were conducted using Gephi 0.8.1 beta (Bastian,

147 Heymann \& Jacomy, 2009). A description of the models using the standard ODD (Overview,

148 Design concepts, Details) protocol and the source code of all models are included in the

149 Supplementary Material.

150

"Single-generation" models

152

We developed these models to test whether two simple strategies of symmetry-based

153 partner choice can reproduce significant emergent features of cooperation in group living

154 animals. In a first model, partner choice was based on an observable arbitrary characteristic of

155 the partners, and agents chose partners in relation to their similarity to themselves. We call this

156 model "Tag Model" (file "Tag_SingleGen_Mdl.nlogo" in the Supplementary Material). This first 
157 model was not spatially explicit, i.e., agents were not set in space. In a second model agents were

158 set in space and chose their partner in relation to their spatial proximity. We call this model

159 "Proximity Model" (file "Prox_SingleGen_Mdl.nlogo" in the Supplementary Material).

160 Agents were created and equipped with a behavioral strategy, which differed in the two

161 models (see below). In the Tag Model, each agent was also assigned a "tag", i.e., a random float

162 number between 0 and 1. In the Proximity Model, each agent was randomly assigned an initial

163 position in a 101 by 101 cells $2 \mathrm{D}$ toroidal space. Note that in both models agents had no memory

164 of past interactions.

165 At each step of the simulation, all agents behaved cooperatively as explained below.

166 First, an agent (the "actor") is randomly selected from the whole population of $N$ agents. Then, a

167 subset of other agents (the "candidates") is randomly extracted among the remaining agents. In

168 the Tag Model, the actor compares its own tag with the tags of the candidates, and directs its

169 cooperative behavior towards the candidate whose tag is the most similar to its own (that is, the

170 actor calculates the absolute differences between its own and the candidates' tags and chose the

171 candidate with the smallest absolute difference). In the Proximity Model, first the actor moves

172 following a simple random walk (i.e., selects a direction of travel randomly, and moves one unit

173 length), then it calculates the distance between itself and the candidates. Finally, the actor directs

174 its cooperative behavior towards the closest candidate (if two or more candidates are equally

175 close, the choice is random between them).

176 All agents in the population go through this sequence at each step of the simulation. The

177 process is asynchronous and the order of agents is randomly chosen at each step of the

178 simulation.

179 Number of candidates was varied systematically as summarized in Table 1. The output of 
180 each simulation was a sociometric matrix of the cooperation given by each agent to each other

181 agent in the population. For each simulation, we calculated the within-subject linear regression

182 between cooperation given and received to/by each other agent. We also calculated two common

183 social network measures (centralization index and modularity) and produced figures representing

184 the social networks of cooperation exchanged between agents.

185

186

\section{"Multi-generation" evolutionary models}

We developed two evolutionary agent based variants of both the Tag Model and the

Proximity Model in order to test whether a strategy of partner choice based on an arbitrary tag or

on spatial proximity can promote the evolution of cooperation. Agents were created and

190

equipped with a behavioral strategy (see below) and other properties (a nongenetically

191 determined tag for the agents in the Tag Model and a position in space for the agents in the

192 Proximity Model). Behavioral interactions had fitness costs and benefits, and the population

193 composition varied generation after generation depending on the evolutionary success of the

194 different strategies agents adopted.

195

196

"Two-strategy" models

197

In a first variant of the evolutionary models, agents were created that adopted one of two different behavioral strategies, choosing cooperative or selfish. Choosing cooperators behaved as described in the single-generation models (i.e., they chose their partner based on its tag in the

200 Tag Model, and based on its spatial proximity in the Proximity Model). Selfish agents never

201 cooperated, but could be the recipient of cooperative behavior by agents adopting the choosing

202 cooperative strategy. At each step of the simulation, each agent behaved according to its own 
203 strategy (files "Tag_MultiGen_Md11.nlogo" and "Prox_MultiGen_Mdl1.nlogo" in the 204 Supplementary Material).

205 Cooperation implied a cost for the actor and a benefit for the recipient. The fitness of

206 each agent was calculated as the difference between the accumulated benefits received and costs

207 incurred during a generation cycle. The selection process consisted in selecting the $20 \%$ of

208 agents with the lowest fitness values at the end of each generation and in removing them from

209 the population. In order to keep population size stable, the $20 \%$ of agents with the highest fitness

210 was made replicate themselves (see Table 2 for details about mutation rates). In the Tag Model,

211 the tag of each agent was initialized at the beginning of each new generation. Note that this

212 corresponds to a model with non overlapping generations in which $20 \%$ of the agents died

213 without reproducing, 60\% died and had one offspring (in the NetLogo code, they remained in the

214 population, but had their tag initialized), and $20 \%$ died and had two offspring. Halving the

215 intensity of selection did not change the results (data not shown). Note also that initializing the

216 tag at each generation implies that the tag is not genetically determined. In the Proximity Model,

217 each agent was assigned a new position in space at the beginning of each new generation. Again,

218 this corresponds to a model with non overlapping generations with no spatial structuring and thus

219 no preferential interaction between kin.

220 We varied the initial proportions of the two strategies, the benefit of receiving

221 cooperation and the constraint on the cooperator's choice (i.e., the number of candidates for the

222 receipt of cooperation). Cost of cooperation did not vary. Details about parameters used in these

223 simulations are shown in Table 2.

224

225 "Continuous probability" models 
227 (choosing cooperative or selfish), agents were characterized by an individual probability of

228 behaving cooperatively (in the vocabulary of evolutionary game theory, these would be "mixed

229 strategies" rather then "fixed strategies") (files "Tag_MultiGen_Md12.nlogo" and

230 "Prox_MultiGen_Md12.nlogo" in the Supplementary Material). At each step of the simulation,

231 each agent could behave as a choosing cooperator (as described above) with probability $\mathrm{P}$ or as a

232 selfish agent with probability 1-P. We created agents and assigned each of them an individual

233 probability $\mathrm{P}$ of behaving as a choosing cooperator. Note that the probability of behaving

234 cooperatively is a fixed characteristic of each individual agent and does not depend on the

235 behavior of other agents. P values were assigned following a normal distribution whose mean

236 and standard deviation was varied as described in Table 2. Other parameters of the simulations

237 are also summarized in Table 2. At the end of each generation, selection and reproduction

238 occurred as described for the previous models.

239

240 RESULTS

241 "Single-generation" models

242 These models evaluated if a population of cooperators adopting symmetry-based

243 reciprocity can reproduce emergent features of cooperation in group living animals. In the Tag

244 Model partner choice was based on an observable characteristic of the partners (the tag), and

245 agents chose partners in relation to their similarity to themselves. In the Proximity Model agents

246 were set in space and chose partners in relation to their spatial proximity.

247 An analysis of the behavior of agents in the Tag Model showed that they reproduced two 248 features of cooperation in group living animals. First, they showed differentiated social 
249 relationships, that is, some pairs cooperated frequently, others less frequently, and others rarely if

250 ever (Fig. 1, top). Second, when correlations between cooperation given and received across

251 pairs were calculated, significant positive relations emerged (Fig. 1, bottom, and Table S2 in the

252 Supplementary Results). When the constraint on the free choice of interactant was progressively

253 decreased, results showed that social network differentiation increased, while the correlation

254 between cooperation given and received first increased and then decreased (Figs. S1 and S2 and

255 Tables S1 and S2 in the Supplementary Results). Also, a progressive formation of clusters of

256 pairs appeared.

257

The analysis of the behavior of agents in the Proximity Model yielded similar results.

Agents showed differentiated social relationships and a positive relation between cooperation

given and received emerged (Fig. 1 and Table S2). Decreasing the constraint on the free choice

of interactant had reduced effects in this model. Social network differentiation increased

moderately, and only a slight reduction in the correlation between cooperation given and received was observed (Figs. S3 and S4 and Tables S1 and S2 in the Supplementary Results).

\section{"Multi-generation" evolutionary models}

We developed these models in order to test the evolutionary success of symmetry-based reciprocity. In these models cooperation had fitness costs (for the actor) and benefits (for the recipient), agents had different behavioral options (cooperate or not) and reproduced at the end of each generation cycle. Selection operated in relation to the fitness (as derived from the accumulated costs and benefits) of each agent. We developed two evolutionary variants of both the Tag Model and the Proximity model. 
272 agents adopting either a cooperative (symmetry-based) or a selfish strategy. Regardless of the

273 parameters of the model, cooperative agents were always outcompeted by selfish agents.

274 Populations initially formed by a majority of cooperative agents were always invaded by selfish

275 agents, and populations initially formed by a majority of selfish agents were never invaded by 276 cooperative agents (Fig. 2 and Figs. S5-S8 in the Supplementary Results).

277 The "two-strategy" variant of the evolutionary Proximity Model adopted the same two

278 discrete strategies, cooperative and selfish. Similarly to what happened in the Tag Model, also in

279 the Proximity Model cooperative agents were never successful (Fig. 3 and Figs. S9-S12 in the

280 Supplementary Results).

281 In the "continuous probability" variant of the evolutionary Tag Model, rather than having

282 two discrete strategies, agents were characterized by an individual probability of behaving

283 (symmetry-based) cooperatively. The results confirmed the selective disadvantage of symmetry-

284 based cooperation. Probability of behaving cooperatively decreased to zero along generations

285 regardless of the parameters of the model and of the initial composition of the population (Fig. 4 286 and Figs. S13-S16 in the Supplementary Results).

287 Similarly, in the "continuous probability" variant of the evolutionary Proximity Model 288 symmetry-based cooperation was never able to survive in the population (Fig. 5 and Figs. S17289 S20 in the Supplementary Results).

290

\section{DISCUSSION}

Our first set of results showed that an agent-based model of symmetry-based reciprocity

293 reproduces aspects of cooperation in group living animals. Our second and more important set of

294 results, however, showed that when reproduction and selection are added to the model, selfish 
295 agents always outcompete agents adopting symmetry-based reciprocity. Although symmetry-

296 based reciprocity appears as a plausible proximate mechanism underlying reciprocal exchanges,

297 it is not evolutionary viable and thus seems to fail as a complete biological explanation of

298 reciprocal cooperation. Indeed, the correspondence between our first set of results and actual

299 cooperative behavior shown by group-living animals appears to be purely phenomenological, as

300 symmetry-based reciprocity cannot evolve and cannot thus underly the behavior of real animals.

301 Before further discussing the implications of these findings, we have to acknowledge

302 their limitations. First, we modeled altruistic behaviors that have a net cost to the actor. It

303 remains to be tested whether symmetry-based reciprocity might play a role in the exchange of

304 mutualistic behaviors, that imply benefits for both the actor and the receiver. Second, simulation

305 studies such as ours cannot in principle be used to demonstrate an impossibility, since they

306 cannot of course cover all theoretically possible combinations of parameters. Therefore, while

307 the results of our study do suggest symmetry-based reciprocity cannot evolve, a conclusive proof

308 would require an analytical demonstration. Our conclusions have therefore to be considered

309 provisional and awaiting analytical confirmation.

310 Our conclusions are coherent with those from the literature on indirect reciprocity, that

311 show how the evolution of cooperation is contingent on the availability of information about the

312 behavior of the potential recipients of cooperation (Nowak \& Sigmund, 1998; Rand \& Nowak,

313 2013). Since in our study the tags used to judge similarity (or proximity) do not encode any

314 information about cooperativeness (either directed to self or to others), selfish cheaters were not

315 avoided and prevailed during evolution. Recently, Rauwolf, Mitchell \& Bryson (2015) showed

316 that in a model of indirect reciprocity, the tendency to interact with individuals sharing similar

317 beliefs (that may be considered analogous to an arbitrary tag) facilitated cooperation. While this 
318 may suggest a role for symmetry in models of indirect reciprocity, its relevance would be limited

319 to humans, as indirect reciprocity seems to be rare in nonhuman animals, possibly because the

320 absence of language constraints the efficient spread of information. Symmetry-based reciprocity,

321 in contrast, has been explicitly proposed (and is typically invoked) to explain reciprocal

322 cooperation in cognitively limited animals (de Waal \& Suchak, 2010), where indirect reciprocity

323 appears to be absent.

324 As already noted, biological phenomena require explanation in terms of four logically

325 separate but interacting aspects. Confusion about these different levels of explanation has most

326 often resulted in assuming unrealistically complex proximate mechanisms for reciprocal

327 cooperation, i.e., in assuming that the delayed return benefits that characterize evolutionary

328 explanations of reciprocity also play a motivational (proximate) role, and thus that reciprocity

329 requires some understanding of future events (de Waal, 2008). Symmetry-based reciprocity

330 resulted to have the opposite shortcoming. It provides a plausible proximate mechanisms for

331 reciprocal cooperation that is not, however, evolutionarily robust.

332 Doubts about the evolutionary viability of symmetry-based reciprocity had already been

333 expressed (Silk, 2005; Schino \& Aureli, 2010). Nevertheless, in the absence of an explicit

334 evolutionary test, symmetry-based reciprocity persisted in the literature and is indeed often

335 considered as a sort of baseline mechanisms that is to be invoked whenever it is not possible to

336 demonstrate more complex mechanisms such as calculated reciprocity (de Waal \& Luttrell,

337 1986; Jaeggi, Stevens \& van Schaik, 2010; de Waal \& Suchak 2010). Decision rules based on

338 interindividual proximity are similarly invoked as a simple/baseline mechanism from which

339 reciprocal cooperation may derive as a byproduct. The need to exclude any effect of

340 interindividual proximity in order to demonstrate "true reciprocity" is thus emphasized (Schino, 
341 Polizzi di Sorrentino \& Tiddi, 2007; Balasubramaniam et al., 2011; Carne, Wiper \& Semple,

342 2011). Having shown its evolutionary weakness, we suggest symmetry-based reciprocity (based

343 on either an arbitrary tag or interindividual proximity) is to be abandoned as a proximate

344 explanation for the occurrence of reciprocity in cognitively unsophisticated animals. Reciprocal

345 cooperation among group living animals that form stable social relationships is most likely

346 supported by emotionally based reciprocity (Schino \& Aureli 2009, 2010, in press), while

347 reciprocity in the absence of stable social relationships (Sella, 1985; Petersen, 1995) does require

348 a simpler mechanisms. Such simpler mechanism, however, cannot be symmetry-based

349 reciprocity given its inability to survive to competition from alternative, selfish strategies.

350 It should be noted here that reciprocal cooperation based on stable social bonds has been

351 sometimes misconceived as an example of symmetry-based reciprocity (de Waal \& Suchak

352 2010). This is incorrect, as the defining characteristic of symmetry-based reciprocity is the

353 absence of any form of bookkeeping of cooperation received. In contrast, emotionally based

354 reciprocity assumes the receipt of cooperative interactions triggers a partner-specific emotional

355 response that translates into a social bond and acts as an emotionally based bookkeeping system

356 of cooperation received. As such, emotionally based reciprocity succeeds as a proximate

357 mechanism for reciprocal cooperation and is also evolutionarily viable (Campennì \& Schino,

358 2014; Evers et al., 2014, 2015, 2016; Puga-Gonzalez, Hoscheid \& Hemelrijk, 2015).

359 The ingroup bias (ethnocentric cooperation) frequently reported in the human literature

360 might be considered as similar to symmetry-based reciprocity. However, human ethnocentric

361 cooperation seems to derive from the need of tight and cooperative within-group relationships in

362 the face of strong inter-group competition (Bowles \& Gintis 2003, 2011) and is therefore

363 different from symmetry-based cooperation as is supposed to operate in cognitively limited 
364 animals. Other models of tag-based (or ethnocentric) cooperation have shown that the

365 evolutionary success of tag-based cooperation requires spatially structured populations with high

366 viscosity, so that agents cooperating with other similarly tagged agents often interact with their

367 own offspring (Hammond \& Axelrod 2006b). Eventually, it is kin selection that can insure the

368 success of tag-based cooperators. In the absence of population viscosity and preferential

369 interaction with kin, tag-based cooperation cannot evolve, coherently with our own results

370 (Axelrod, Ammond \& Grafen, 2004; Hammond \& Axelrod 2006a). It should be noted that in

371 these models the tag used to guide decisions about cooperation is genetically determined, and

372 thus coevolves with cooperation. In our model, on the contrary, the tag is not genetically

373 determined and cannot evolve. It should also be noted that these previous models of tag-based

374 cooperation were always partner control models in which cooperative interactions were included

375 as one-shot prisoner dilemmas. In contrast, our models are partner choice models in which

376 obligate cooperators chose the recipient of their cooperation on the basis of some defined rule

377 (Noë, 2001; Campennì \& Schino, 2014). Our preference for a partner choice model with a non

378 genetically determined tag derived from the need to model symmetry-based reciprocity,

379 particularly as acting in group living animals.

$380 \quad$ Previous agent based models of cooperative exchanges in group living animals had

381 emphasized the role of interindividual proximity in producing patterns of group distribution of

382 cooperative exchanges that reproduce "apparent" reciprocity (Puga-Gonzalez, Hildenbrandt \&

383 Hemelrijk, 2009; Hemelrijk \& Puga-Gonzalez 2012). Our results confirmed that a spatially

384 structured model in which decisions about cooperation are made according to simple

385 interindividual proximity can generate patterns of cooperative exchanges that are similar to those

386 observed in group living animals. Our models, however, went one step further by demonstrating 
387 that such simple decision rule is not evolutionarily stable.

388 In conclusion, the results of our study highlight how hypotheses about the proximate

389 mechanisms underlying behavior cannot leave aside considerations about their evolvability. The

390 integration of tests of proximate determinants and of ultimate functions provides a more

391 compelling test of biological hypotheses and should be recommended whenever possible (Akçay

392 et al., 2009). To the extent that the results of our simulations can be generalized (and awaiting

393 their analytical confirmation), symmetry-based reciprocity seems to fail such a double test, and

394 should therefore be abandoned as a hypothetical proximate mechanism supporting animal

395 cooperation. It is left to the ingenuity of students of animal behavior to hypothesize a new

396 biologically plausible proximate mechanism that could support reciprocity in cognitively

397 unsophisticated animals that do not form stable social relationships (Schino \& Aureli, in press). 398

399 ACKNOWLEDGEMENTS

We thank Federico Cecconi for helpful discussions. 


\section{REFERENCES}

403

404

405

406

407

408

409

410

411

412

413

414

415

416

417

418

419

420

421

422

423

424

Akçay E, Van Cleve J, Feldman MW, Roughgarden, J. 2009. A theory for the evolution of other-regard integrating proximate and ultimate perspectives. Proc Natl Acad Sci U S A 106: 19061-19066.

Axelrod R, Ammond RA, Grafen, A.2004. Altruism via kin-selection strategies that rely on artibrary tags with which they coevolve. Evolution 58: 1833-1838.

Balasubramaniam KN, Berman CM, Ogawa H, Li J. 2011. Using biological markets principles to examine patterns of grooming exchange in Macaca thibetana. Am J Primatol 73: $1269-79$.

Bastian M, Heymann S, Jacomy M. 2009. Gephi: an open source software for exploring and manipulating networks. In: Proceedings of the Third International ICWSM Conference, pp $361-362$.

Bowles S, Gintis H. 2003. Origins of human cooperation. In: Hammerstein P, (ed.) Genetic and Cultural Evolution of Cooperation. MIT Press, Boston, pp 429-443.

Bowles S, Gintis H. 2011. A Cooperative Species: Human Reciprocity and Its Evolution. Princeton University Press, Princeton.

Brosnan SF, de Waal FBM. 2002. A proximate perspective on reciprocal altruism. Hum Nat 13: $129-152$.

Bryson JJ, Ando Y, Lehmann H. 2007. Agent-based modelling as scientific method: a case study analysing primate social behaviour. Phyl Trans R Soc B 362: 1685-98.

Bull JJ, Rice WR. 1991. Distinguishing mechanisms for the evolution of cooperation. $J$ Theor Biol 149: 63-74.

Campennì M, Schino G. 2014. Partner choice promotes cooperation: The two faces of testing 
with agent-based models. J Theor Biol 344: 49-55.

426 Carne C, Wiper S, Semple S. 2011. Reciprocation and interchange of grooming, agonistic

427 support, feeding tolerance, and aggression in semi-free-ranging Barbary macaques. Am $J$

428 Primatol 73: 1127-1133.

429

430

431

432

433

434

435

436

437

438

439

440

441

442

443

444

de Waal FBM. 2000. Attitudinal reciprocity in food sharing among brown capuchin monkeys. Anim Behav 60: 253-261.

de Waal FBM. 2008. Putting the altruism back into altruism: the evolution of empathy. Annu Rev Psychol 59: 279-300.

de Waal FBM, Luttrell LM. 1988. Mechanisms of social reciprocity in three primate species: symmetrical relationships characteristics or cognition? Ethol Sociobiol 9: 101-118.

de Waal FBM, Luttrell LM. 1986. The similarity principle underlying social bonding among female rhesus monkeys. Folia Primatol 46: 215-234.

de Waal FBM, Suchak M. 2010. Prosocial primates: selfish and unselfish motivations. Phyl Trans $R$ Soc B 365: 2711-22.

Evers E, de Vries H, Spruijt BM, Sterck EHM. 2014. The EMO-model: an agent-based model of primate social behavior regulated by two emotional dimensions, anxiety-FEAR and satisfaction-LIKE. PLoS One 9: e87955.

Evers E, de Vries H, Spruijt BM, Sterck EHM. 2015. Emotional bookkeeping and high partner selectivity are necessary for the emergence of partner-specific reciprocal affiliation in an agent-based model of primate groups. PLoS One 10: e0118921.

Evers E, de Vries H, Spruijt BM, Sterck EHM. 2016. Intermediate-term emotional bookkeeping is necessary for long-term reciprocal grooming partner preferences in an agent-based model of macaque groups. PeerJ 4, e1488. 
448 Fawcett TW, Marshall JAR, Higginson AD. 2015. The Evolution of mechanisms underlying 449 behaviour. Current Zoology 61: 221-225.

450 Franz M, Schülke O, Ostner J. 2013. Rapid evolution of cooperation in group-living animals. $451 \quad$ BMC Evol Biol 13: 235.

452 Gardner A, West SA. 2010. Greenbeards. Evolution 64: 25-38.

453 Gould SJ, Lewontin RC. 1979. The spandrels of San Marco and the Panglossian paradigm: a $454 \quad$ critique of the adaptationist programme. Proc $R$ Soc B 205: 581-598.

455 Hamilton WD. 1964. The genetical evolution of social behaviour. I. J Theor Biol 7: 1-16.

456 Hammond RA, Axelrod R. 2006a. Evolution of contingent altruism when cooperation is 457 expensive. Theor Popul Biol 69: 333-8.

458 Hammond RA, Axelrod R. 2006b. The evolution of ethnocentrism. J Conflict Resolut 50: 926459936.

460 Hemelrijk CK, Puga-Gonzalez I. 2012. An individual-oriented model on the emergence of $461 \quad$ support in fights, its reciprocation and exchange. PLoS One 7: e37271.

462 Hofmann HA, Beery AK, Blumstein DT, Couzin ID, Earley RL, Hayes LD, Hurd PL, 463 Lacey EA, Phelps SM, Solomon NG, Taborsky M, Young LJ, Rubenstein DR. 2014. 464 An evolutionary framework for studying mechanisms of social behavior. Trends Ecol Evol 465 29: 581-589.

Holekamp KE, Swanson EM, van Meter PE. 2013. Developmental constraints on behavioural $467 \quad$ flexibility. Phil Trans $R$ Soc B 368: 20120350.

468 Jaeggi, AV, Stevens, JMG, van Schaik CP. 2010. Tolerant food sharing and reciprocity is 469 precluded by despotism among bonobos but not chimpanzees. Am J Phys Anthropol 51: 41$470 \quad 51$. 
471 Lehmann L, Keller L. 2006. The evolution of cooperation and altruism: a general framework $472 \quad$ and a classification of models. J Evol Biol 19: 1365-1376.

473 Mayr E. 1961. Cause and effect in biology. Science 134: 1501-1506.

474 Mayr E. 1982. The Growth of Biological Thought. Harvard University Press, Cambridge.

475 Noë R. 2001. Biological markets: partner choice as the driving force behind the evolution of 476 mutualism. In: Noë R, van Hooff JARAM, Hammerstein P (eds) Economics in Nature. 477 Cambridge Universtiy Press, Cambridge, pp 93-118.

478 Noë R. 2006. Cooperation experiments: coordination through communication versus acting apart $479 \quad$ together. Anim Behav 71: 1-18.

Nowak MA, Sigmund K. 1998. Evolution of indirect reciprocity by image scoring. Nature, 393, 481 $573-577$.

482 Nowak MA, Sigmund K. 2005. Evolution of indirect reciprocity. Nature, 437: 1291-1298.

483 Petersen CW. 1995. Reproductive behavior, egg trading, and correlates of male mating success 484 in the simultaneous hermaphrodite, Serranus tabacarius. Environ Biol Fishes 43: 351-361.

485

486

Puga-Gonzalez I, Hildenbrandt H, Hemelrijk CK. 2009. Emergent patterns of social affiliation in primates, a model. PLoS Comput Biol 5: e1000630.

Puga-Gonzalez I, Hoscheid A, Hemelrijk CK. 2015. Friendship, reciprocation, and interchange in an individual-based model. Behav Ecol Sociobiol 69: 383-394.

R Development Core Team. 2012. R: A Language and Environment for Statistical Computing. R Foundation for Statistical Computing, Vienna, Austria, "http://www.R-project.org/".

Rand DG \& Nowak MA. 2013. Human cooperation. Trends Cognit Sci, 17, 413-425.

Rauwolf P, Mitchell D, Bryson JJ. 2015. Value homophily benefits cooperation but motivates employing incorrect social information. J Theor Biol 367, 246-261. 
494 Riolo RL, Cohen MD, Axelrod R. 2001. Evolution of cooperation without reciprocity. Nature 495 414: 441-443.

496 Roberts G. 1998. Competitive altruism: from reciprocity to the handicap principle. Proc $R$ Soc $B$ $497 \quad$ 265: 427-431.

Rousset F, Roze D. 2007. Constraints on the origin and maintenance of genetic kin recognition. Evolution 61: 2320-2330.

Schino G, Aureli F. 2008. Grooming reciprocation among female primates: a meta-analysis. Biol Lett 4: 9-11.

Schino G, Aureli F. 2009. Reciprocal altruism in primates: partner choice, cognition, and emotions. Adv Study Behav 39: 45-69.

Schino G, Aureli F. 2010. Primate reciprocity and its cognitive requirements. Evol Anthropol 19: $130-135$.

Schino G, Aureli F. 2010. in press. Reciprocity in group-living animals: partner control versus partner choice. Biol Rev.

Schino G, Polizzi di Sorrentino E, Tiddi B. 2007. Grooming and coalitions in Japanese Macaques (Macaca fuscata): partner choice and the time frame of reciprocation. J Comp Psychol 121: 181-188.

Sella G. 1985. Reciprocal egg trading and brood care in a hermaphroditic polychaete worm. Anim Behav 33: 938-944.

Seyfarth RM, Cheney DL. 2012. The evolutionary origins of friendship. Annu Rev Psychol 63: $153-177$.

Silk JB. 2005. The evolution of cooperation in primate groups. In: Gintis H, Bowles S, Boyd R, Fehr E, (eds) Moral Sentiments and Material Interests: On the Foundations of Cooperation 
517 in Economic Life. MIT Press, Boston, pp 43-73.

518 Stevens JR, Hauser MD. 2004. Why be nice? Psychological constraints on the evolution of 519 cooperation. Trends Cogn Sci 8: 60-65.

520 Timbergen N. 1963. On aims and methods of ethology. Z Tierpsychol 20: 410-433.

521 Trivers RL. 1971. The evolution of reciprocal altruism. Q Rev Biol 46: 35-57.

522 Trivers RL. 2006. Reciprocal altruism: 30 years later. In: Kappeler PM, van Schaik CP (eds)

523 Cooperation in Primates and Humans. Springer, Berlin, pp 67-83.

524 Wilensky, U. 1999. NetLogo "http://cclnorthwesternedu/netlogo/". Center for Connected

525 Learning and Computer-Based Modeling, Northwestern University, Evanston, IL.

526 
531 Table 1. Parameters used to run the "single-generation" models.

\begin{tabular}{|l|l|}
\hline Parameter & Values \\
\hline Population size (N of agents) & 50 \\
\hline Candidates for the interaction (N of agents) & $2,10,25,49$ \\
\hline Number of steps per simulation & 1000 \\
\hline Number of simulations (replicates) & 100 \\
\hline
\end{tabular}

533

534 
Table 2. Parameters used to run the evolutionary models.

536

\begin{tabular}{|l|l|l|}
\hline Parameter & \multicolumn{1}{|l|}{ Values } \\
\hline & "Two-strategy" model & "Continuous probability" model \\
\hline Population size (N of agents) & 50 & 50 \\
\hline Candidates for the interaction (N of agents) & $2,10,25,49$ & $2,10,25,49$ \\
\hline Number of steps per generation & 1000 & 1000 \\
\hline Number of generation per simulation & 50 & 200 \\
\hline Number of simulations (replicates) & 30 & 30 \\
\hline Benefit of receiving cooperation (fitness units) & $1.1,2,5,50$ & $1.1,2,5,50$ \\
\hline Cost of cooperation (fitness units) & 1 & 1 \\
\hline Number of strategies & $2($ choosing cooperative, & 1 (variable prob. of behaving \\
& selfish) & cooperatively) \\
\hline Initial strategy ratio or distribution of prob. of behaving & $45 / 5,25 / 25,5 / 45$ & $0.05 \pm 0.05,0.5 \pm 0.35,0.95 \pm 0.05$ \\
\hline cooperatively & & 0.1 \\
\hline Mutation rate & switch strategy & \pm 0.2 \\
\hline Mutation effect & 0.2 & 0.2 \\
\hline Proportion of agents selected for reproduction & & 0.1 \\
\hline
\end{tabular}




\section{FIGURE LEGENDS}

539 Fig. 1. The distribution of cooperative interactions among agents in the Tag and Proximity Models. Top: Networks of cooperation exchanged. Bottom: Cooperation given in relation make their choice between 10 randomly selected other agents. See the Supplementary Results for the effects of varying additional parameters.

544 Fig. 2. The evolution of symmetry-based reciprocity in the "two-strategy" Tag Model. Symmetry-based cooperators: black dots; selfish: grey dots. Populations varied in relation

Fig. 3. The evolution of symmetry-based reciprocity in the "two-strategy" Proximity Model. effects of varying additional parameters. to their initial composition and in the fitness benefits of receiving cooperation. Cost of cooperation for the actor was always 1 fitness unit. Choosing cooperators could make their choice among 10 randomly selected other agents. See the Supplementary Results for the Symmetry-based cooperators: black dots; selfish: grey dots. Populations varied in relation to their initial composition and in the fitness benefits of receiving cooperation. Cost of cooperation for the actor was always 1 fitness unit. Choosing cooperators could make their choice among 10 randomly selected other agents. See the Supplementary Results for the effects of varying additional parameters.

556 Fig. 4. The evolution of symmetry-based reciprocity in the "continuous probability" Tag Model. Populations varied in relation to their initial composition and in the fitness benefits of receiving cooperation. Cost of cooperation for the actor was always 1 fitness unit. Agents could make their choice among 10 randomly selected other agents. See the Supplementary Results for the effects of varying additional parameters. 
561 Fig. 5. The evolution of symmetry-based reciprocity in the "continuous probability" Proximity

Model. Populations varied in relation to their initial composition and in the fitness benefits of receiving cooperation. Cost of cooperation for the actor was always 1 fitness unit. Agents could make their choice among 10 randomly selected other agents. See the Supplementary Results for the effects of varying additional parameters. 
568

569

570

571

572

573

574

575

576

577

578

579

580

\section{Proximity Model}
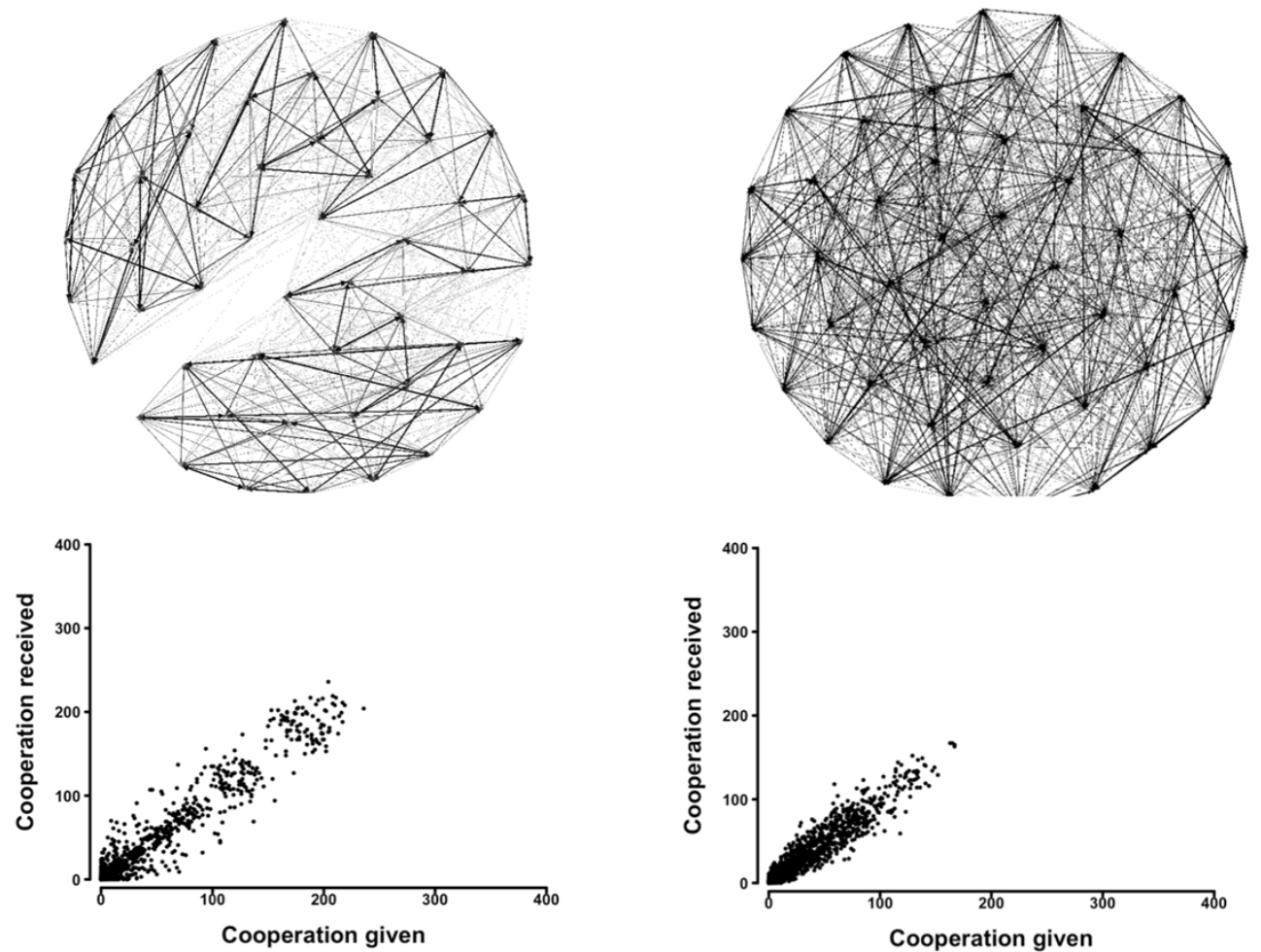

Fig. 1 
581

582

583

584
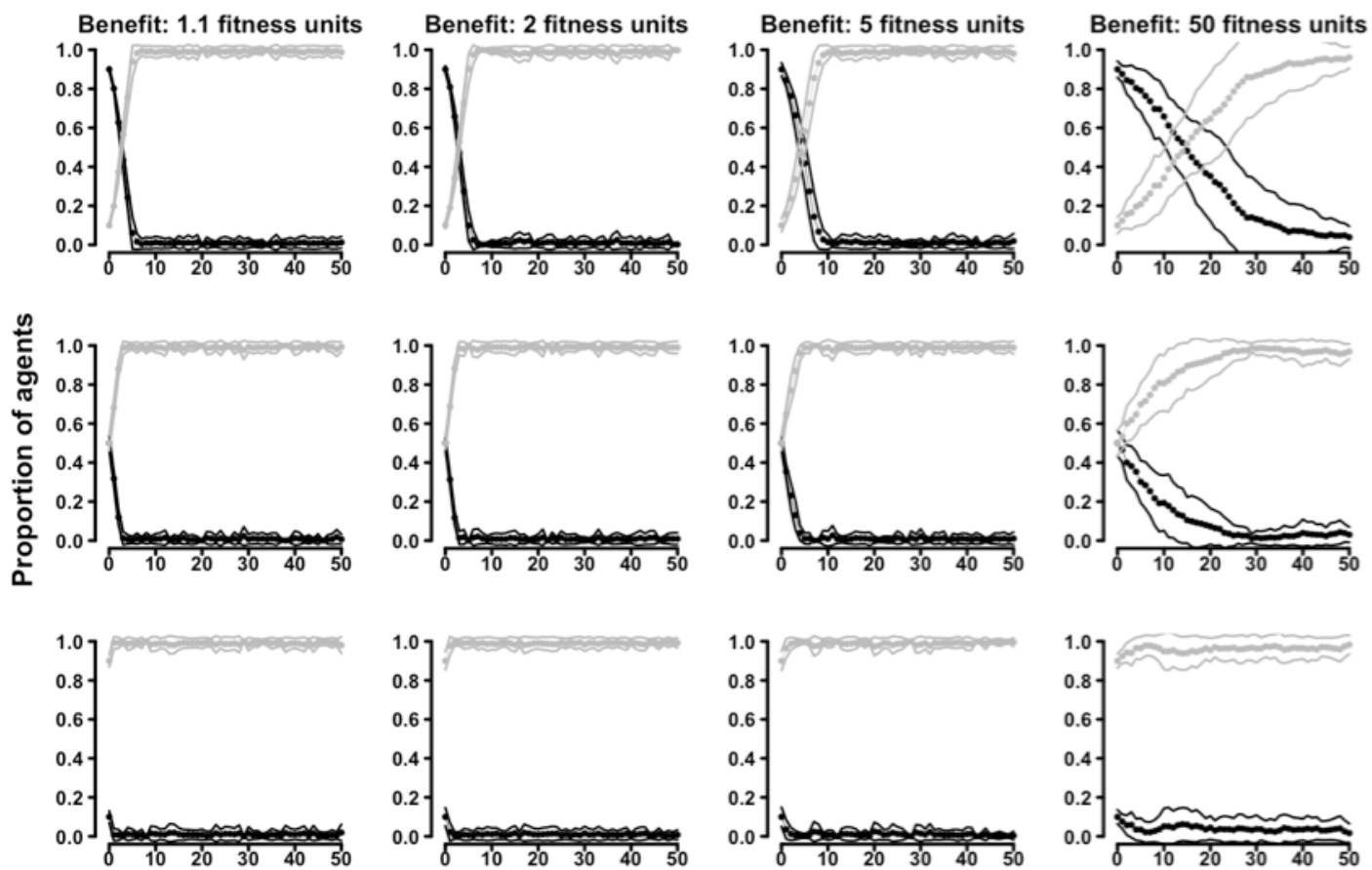

585

586

587

588

589

590

591

592

593
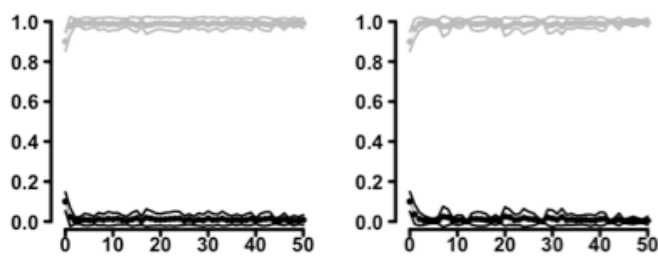

Time (generation)

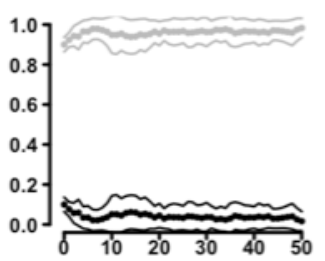

Fig. 2 
594

595

596

597
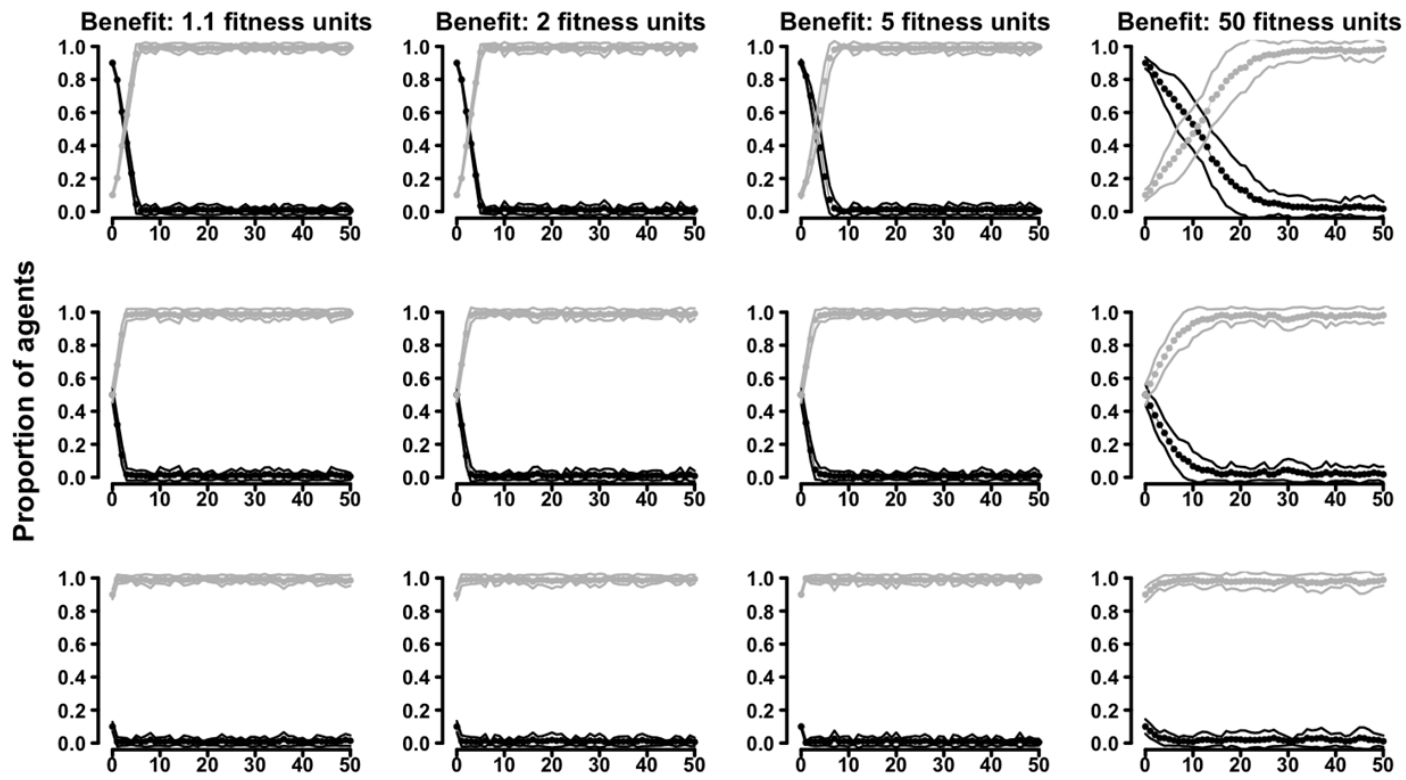

Time (generation)

601

602

603

604

Fig. 3

605

606 
607

608

609

610

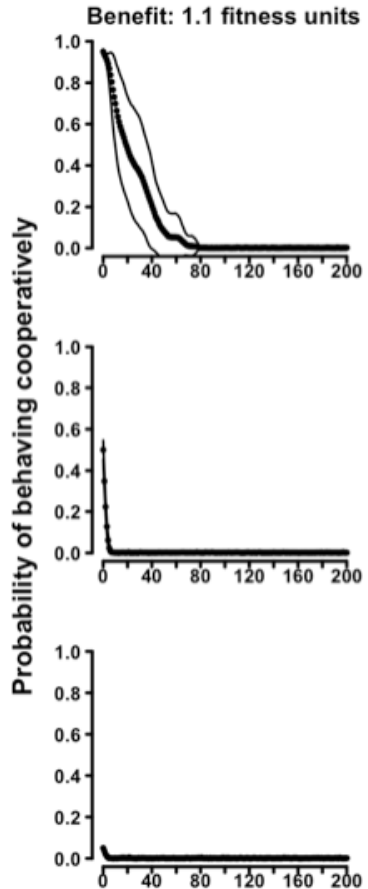

611

612

613

614

615

616

617

618

619
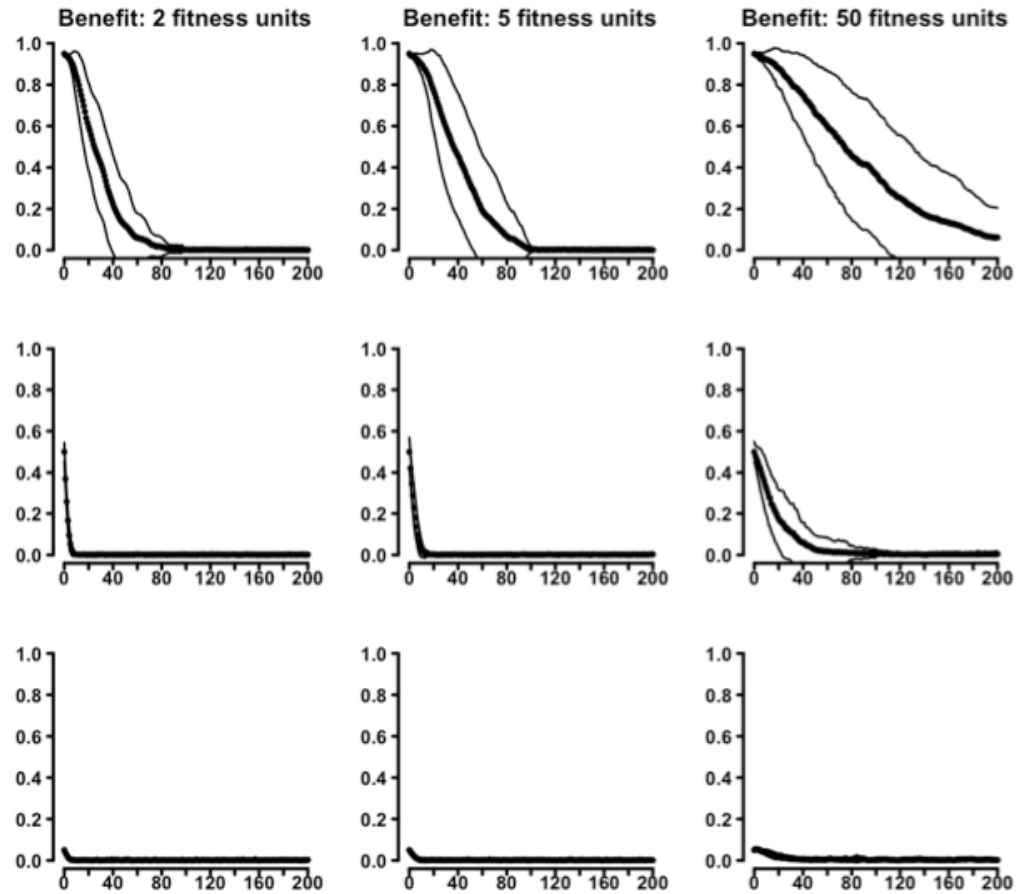

Fig. 4 
623
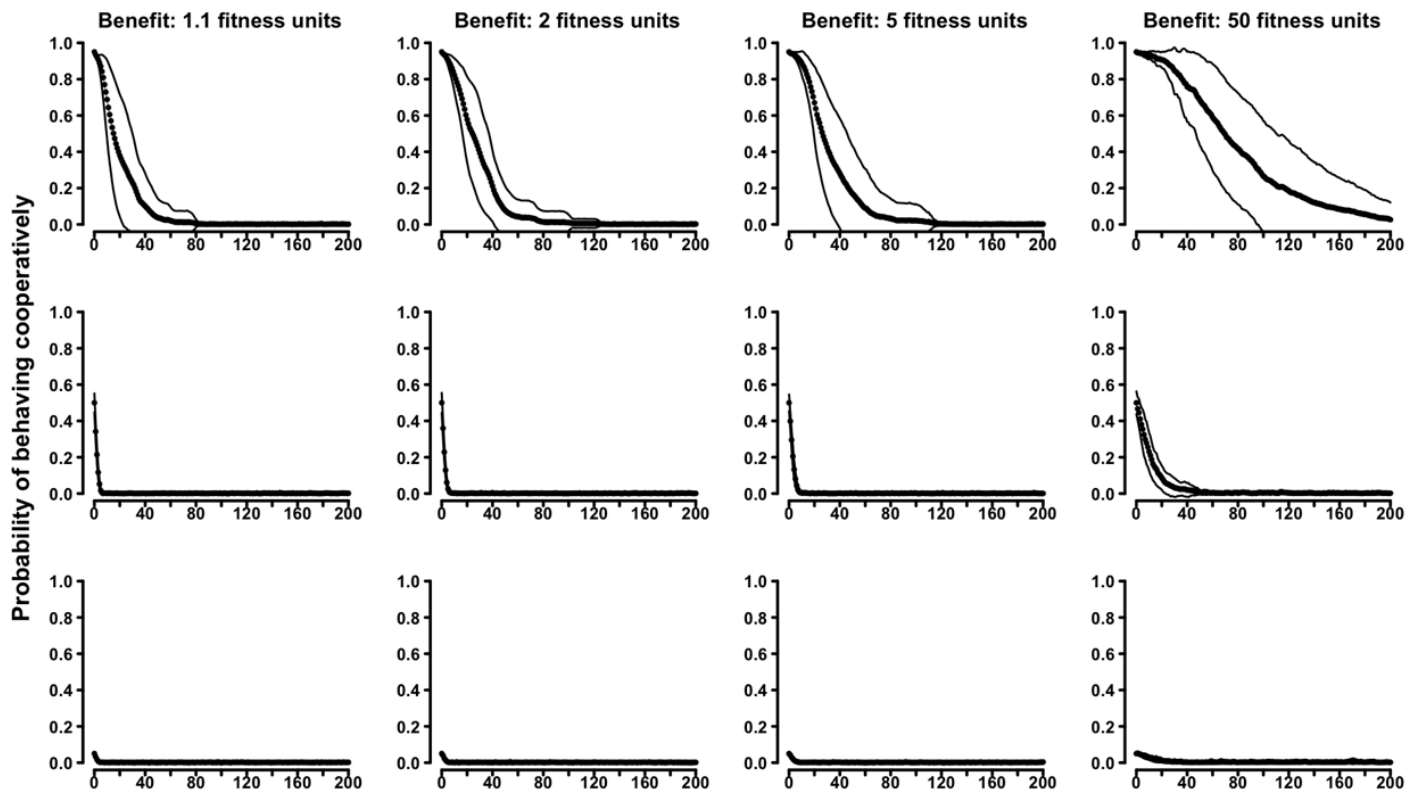

624
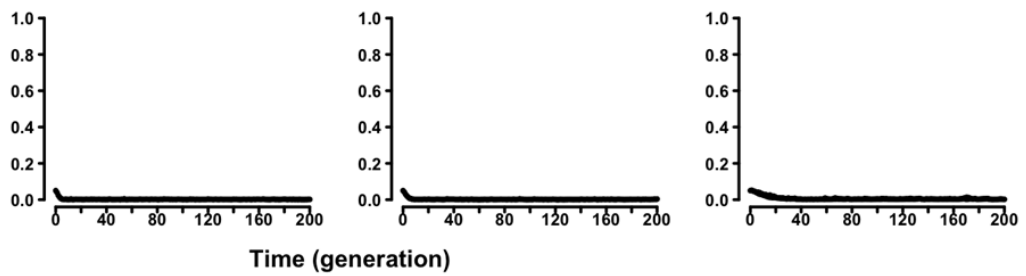

625

626

627

628

629

630

Fig. 5

631

632 